\title{
V.
}

Aus dem pharmakologisehen Institut der Universität Wien.

\section{Über den infravitalen Eiweißabbau in der Leber sensibilisierter Tiere und dessen Beeinflussung durch die Milz.}

\author{
Von \\ Masakadzu Hashimoto (Osaka) und Ernst P. Pick. \\ Mit 1 Figur.
}

Durch 1) zahlreiche Untersuchungen der letzten Jahre wurde festgestellt, daß artfremdes Eiweiß, welches mit Umgehung des Digestionstraktes in den tierischen Körper eingeführt wird, von dem Organismus teils unverändert ausgeschieden, teils jedoch in niedere $\mathrm{N}$-haltige Spaltprodukte abgebaut wird (Friedemann und Is aak ${ }^{2}$ ), Heilner ${ }^{3}$ ), Lomme14), Oppenheimer ${ }^{5}$ ), Cramer ${ }^{6}$ ), Michaelis und Rona ${ }^{7}$ ), de Waele und Vandevelde ${ }^{8}$ ), Sehittenhelm und Weichardt) ${ }^{\%}$. Man schloß aus dieser Tatsache, daß der tierische Organismus auch

1) Einige der nachfolgenden Untersuchungsergebnisse wurden bereits in der Diskussion zu Abderhaldens Vortrag am IX. internat. Physiologenkongreß in Groningen 2.-6. Sept. 1913 erwilint, sowie in Zentralblatt für Physiologie Bd. 27, Nr. 16, vorläufig mitgeteilt.

2) Friedemann, U. und Isaak, S., Zeitschr. f. exper. Path. u. Ther. Bd. I, S. 513, 1905; Bd. III, S. 209, 1906 ; Bd. IV, S. 830, 1907.

3) Heilnèr, E., Zeitsehr. f. Biolog. Bd. 50, S. 26, 1907 und Bd. 52, 1908, ferner Bd. 58, S. 333, 1912.

4) Lommel, F., Arch. f. exper. Path. u. Pharmakol. Bd. 58, S. 50, 1907.

5) Oppenheimer, C., Hofmeisters Beiträge Bd. 4, S. 263, 1903.

6) Cramer, W., Journ. of physiol. Vol. 37, p. 146. 1908.

7) Michaelis und Rona, Pfügers Arch. Bd. 121, S. 163, 1908 u. Bd. 123, S. 406, 1908.

8) De Waele, H. und Vandevelde, A. J. J., Biochem. Zeitschr. Bd. 30, S. 227, 1911.

9) Schittenhelm, A. und Weichardt, W., Zeitschr. f. exper. Path. und Ther. Bd. 11, S. 68, 1912. 
parenteral zugeführtes Eiweiß zu assimilieren vermag. Diese Ansicht gewann eine besondere Stütze durch den bedeutsamen Befund von Abderhalden und Pincussohn1), daß Blutplasma oder Blutserum von Tieren, welche mit artfremden oder blutfremden Eiweißkörpern vorbehandelt worden waren, die fermentative Fähigkeit gewann, Eiweißkörper und deren Spaltprodukte auch außerhalb des Körpers abzubauen.

Die durchgreifenden Änderungen, welche zweifellos der Organismus durch die genannte Form der Eiweißdarreichung in seinen fermentativen Eigenschaften erfährt, treten am augenfälligsten im anaphylaktischen Shock eiweißvorbehandelter Tiere hervor; es war naheliegend, beide Erscheinungen, Eiweißabbau und Shock, in einen ursächlichen Zusammenhang zu bringen, zumal eine Reihe experimentell erhobener Tatsachen (Befund von Eiweißspaltprodukten im Blute anaphylaktischer Tiere, Erzeugung charakteristischer Vergiftungssymptome durch Eiweißabbauprodukte) in diesem Sinne sich deuten ließ. Neuere Beobachtungen scheinen indessen daranf binzuweisen, daß nicht allein das Blut und dessen Fermente an der Umstimmung des Körpers Anteil haben, sondern daß wahrseheinlich in den versehiedensten Zellen des Organismus hochgradige biolo. gische Änderungen durch die Vorbehandlung mit Eiweiß (Sensibilisierung) vor sich gehen, welche sich in der verschiedensten Weise, meist aber im Sinne der Beschleunigung und Steigerung des Reaktionsablaufes mannigfacher Lebensprozesse äußern. Hier wäre vor allem zu erinnern an die intensive Steigerung der spezifischen Erregbarkeit der isolierten glatten Muskulatur des Darmes, des Uterus und der Bronchien von sensibilisierten Meerschweinchen und Katzen, wie sie in höchst interessanten Versuchen von Schultz und besonders von Dale $\left.{ }^{3}\right)$ festgestellt wurde. Versuche von Yamanouchi ${ }^{4}$ ), der die Nerven (Ischiadicus) vorbehandelter Kaninchen untersuchte, ergaben strenge spezifische Änderungen ihrer Erregbarkeit. Auch der

1) Abderhalden, E. u. Pincussohn, L., Zeitschr. f. physiolog. Chemie Bd. 61, S. 200, 1909; Bd. 62, S. 243, 1909; Bd. 64, S. 100, 1910 sowie Abderhalden, E. und Weichardt, W., Bd. 62, S. 120, 1909. Siehe auch die Zusammenstellnng der einschlägigen Literatur bei Abderhalden, Schutzfermente des tierischen Organismus, Berlin 1912; sowie W. Caspari im Handbuch der Biochemie, Ergänzungsband S. 78, 1913.

2. Schultz, W. H, Journ of pharmac. and exper. therap Vol. 1, p. 549, 1910 und Vol. 2, p. 221, 1910; Vol. 3, p. 299, 1912; ferner Hygienic Laboratory Bulletin Nr. 80, 1912.

3) Dale, H. H, Journ. of pharmac. and exper. therap. Vol. IV, p. 167, 1913.

4) Yamanouchi, T., Annales de l'Instit. Pasteur. T. 23, p. 577, 1909. 
bemerkenswerte Abfall der Körpertemperatur, wie ihn H. Pfeiffer ${ }^{1}$ ) fand, sowie die Herabsetzung des Gesamtstoffwechsels (Loening ${ }^{2}$ ) im anaphylaktischen Shock weisen auf die große Empfindlichkeit der 'verschiedensten Körperzellen sensibilisierter Tiere gegenüber spezifischen Reizen hin.

Die Beeinflussung anderweitiger zeilulärer Prozesse durch die Eiweißsensibilisierung schien daher nicht aussichtslos und wir wendeten unsere Aufmerksamkeit vor allem der Leber zu, da diese Drüse durch ihren maßgebenden Anteil am Gesamtstoffwechsel von vornherein in den Mittelpunkt des Geschehens bei eventuellen Änderungen des fermentativen Stoffwechsels gerïckt ist. Zudem liegen über die Bedeutung der Leber beim Auslösen des anaphylaktischen Shockes wichtige Angaben von Manwaring ${ }^{3}$ ) sowie von Voegtlin und Bernheim ${ }^{4}$ ) vor, welche neuestens auch von Dale ${ }^{5}$ ) bestätigt worden sind; sie zeigen, daß bei Leberausschaltung ans dem Kreislauf beim Hunde der anaphylaktische Shock nicht erzeugt werden kann; ob anch bei der Anaphylaxie des Meerschweinchens die Leber eine Rolle spielt, ist unentschieden, da schon durch die Sensibilisierung der Bronchialmuskulatur unabhängig von der Leber (Dale) rasch der tödliche Lungenkrampf herbejgeführt wird.

Unsere erste Aufgabe bestand darin, die chemischen Vorgänge während der Eiweißsensibilisierung in der Leber zu studieren, und wir untersuchten zunächst mit Rücksicht auf die Möglichkeit, daß in der Leber sensibilisierter Tiere proteolytische Vorgänge sich abspielen, die in der Norm entweder ïberhaupt nicht oder nur in beschränktem Maße stattfinden, ob die Menge der stickstoffhaltigen Abbauprodukte intravital in der Leber normaler und eiweiBsensibilisierter Tiere einen merklichen Unterschied aufweist. Zu diesem Zwecke wurden Meerschweinchen als die fur die Eiweißsensibilisierung empfindlichsten Tiere mit 0,5 cem nativem Pferdeserum subkutan injiziert, 3-68 Tage nach dieser Vorbehandlung durch Durchschneidung beider Karotiden entblutet und die sofort körperwarm entnommene, blasse Leber verarbeitet. Als Kontrolle diente die Leber unvorbehandelter Meerschweinchen, die in gleicher Weise getötet wurden. Die Tiere besaßen in der Regel das Gewicht von 250-300 g und wurden mit

1) Pfeiffer, H., Wiener klin. Wochenschr. 1909, Nr. 1, 36 u. 40; ferner Pfeiffer, H. und Mita, S., Zeitschr. f. Immunitätsforschung Bd. 4, S. 410, 1909 and Bd. 6, S. 18, 1910.

2. Loening, F., Arch. f. exper. Path. u. Pharmakol. Bd. 66, S. 84, 1911.

3) Manwaring, W. H., Zeitschr. f. Immunitätsforschung Bd. 8, S. 1, 1910.

4) Voegtlin C. and Bernheim, Journ. of pharmacol. and exper. therap. 1911, Nr. 6.

5) Dale, H. H., a. a. 0. 
gleichem Futter - Hafer mit Kohlblättern - genährt; die Freßlust der vorbehaudelten Tiere unterschied sich in nichts von den normalen.

Die Leber jedes einzelnen Tieres gelangte für sich in folgender Weise zur Untersuchung: das Organ wurde nach Entfernung der Ligamente obne Waschen in einer kleinen Reibschale mit dem Pistill zu einem feinen, halbfliussigen Brei zerrieben und durch ein trockenes feines Metallsieb getrieben; der erhaltene sirupöse Organbrei wurde in drei Portionen geteilt und jede in einem größeren, etwa 20-30 ccm fassenden Wägegläschen genau abgewogen; das Gewicht einer jeden Portion betrug etwa $1,5-2 \mathrm{~g}$.

Wäbrend zwei Portionen sofort zur Bestimmung des Gesamtstickstoffs und des Stickstoffs der nicht durch Hitze koagulablen Stickstoffkörper (unkoagulabler Stickstoff) herangezogen wurden, wurde die dritte Portion im Wägegläschen nach Zusatz von einer kleinen Menge 0,9\% iger Kochsalzlösung und $2 \mathrm{ccm}$ Toluols mit einem Glasstäbchen bis zur gleichmäßigen Verteilung vorsichtig durchgerührt, der Glasstab mit der Kochsalzlösung abgespuilt und das Ganze bis zu dem zehnfachen Volumen des Gewichtes der betreffenden Leberportion mit $0,9 \%$ iger Kochsalzlösung aufgefüllt; diese Probe wurde im geschlossenen Wägegläschen im Brutschrank bei $37^{\circ}$ der Autolyse iiberlassen.

Der Gesamtstickstoff der ersten Portion wurde nach Kjeldahl bestimmt; die Entleerung des Leberbreies aus dem Wägegläschen in den Zersetzungskolben gelingt leicht mit Hilfe eines feinen Glasstäbchens, wobei die letzten Reste des Breies quantitativ mit destilliertem Wasser in den Kolben gespuilt werden. Die Bestimmung des unkoagulablen Stickstoffs der zweiten Portion wurde derart vorgenommen, daß der abgewogene Leberbrei aus dem Wägegläschen quantitativ in ein dünnwandiges Becherglas gespült und darin der auf etwa $60-70 \mathrm{~cm}$ mit destilliertem Wasser verdiunnte Organbrei nach Zusatz von zwei Messerspitzen festen Kochsalzes und einiger Tropfen Essigsäure (bis zur schwachsauren Reaktion der Flüssigkeit) koaguliert; die Koagulation wurde stets im kochenden Wasserbad so lange vorgenommen, bis das geronnene Eiweiß in groben Flocken am Boden des Becherglases sich sammelte, während die überstehende Flüssigkeit völlig klar blieb; die letztere war leicht hellgelb gefärbt und durfte keine Spur einer Opaleszenz zeigen 1 ); die hierza nötige

1) Kontrollversuche haben im übrigen ergeben, daß selbst leicht opale Flüssigkeiten gegenüber völlig klaren Lösungen keinen wesentlichen Unterschied im N-Gehalt aufwiesen, so daß die Opaleszenz nicht auf unvollständiger Eiweißkoagulation, sondern wahrscheinlich auf dem Glykogengehalte der Leber beruht. 
Erhitzungsdauer betrug 20-35 Minuten. Dieses alte Salkowskische Koagulationsverfahren lieferte uns bei sorgfältigem Arbeiten durchaus zuverlässige und gleichmäßige Werte und bot gegenüber anderen Koagulationsmethoden den Vorteil, daB Verluste an unkoagulablem Eiweiß-N, die bei anderen Verfahren durch Mitgerissenwerden in kolloidale Niederschläge oder Aussalzen eintreten können, vermieden wurden. Die koagulierte Flïssigkeit wurde in einem Meßkolben auf $100 \mathrm{ccm}$ aufgefüllt, filtriert und je $25 \mathrm{ccm}$ des Filtrates zur N-Bestimmung nach Kjeldahl verwendet; alle Bestimmungen wurden doppelt ausgeführt; die Titration erfolgte mit $1 / 10 \mathrm{NH}_{2} \mathrm{SO}_{4}$ und $1 / 10 \mathrm{~N} \mathrm{NaOH}$ unter Benutzung von Cochenille als Indikator. Coccidienkranke Lebern, denen wir hier und da begegneten, wurden ron der Untersuchung ausgeschlossen, da es sich zeigte, daß dieselben von vornherein einen höberen Gehalt an unkoagulierbaren N-haltigen Stoffen aufwiesen.

Die Verarbeitung der der Antolyse unterworfenen dritten Portion soll in einer späteren Mitteilung gesondert besprochen werden.

\section{I. Über das Verhältnis des unkoagulablen Stickstoffs zu} dem Gesamtstickstoff der normalen Meerschweinchenleber.

Die in der eben geschilderten Weise durchgeführte Verarbeitung der Lebern von acht etwa $250 \mathrm{~g}$ schweren, frisch entbluteten Meerschweinchen, die keinerlei Vorbehandlung erfahren haben, ergab nachfolgende Resultate. Da das Durchschnittsgewicht einer Meer-

Tabelle 1.

\begin{tabular}{|c|c|c|c|c|c|}
\hline 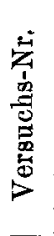 & $\begin{array}{l}\text { Gesamtstick- } \\
\text { stoff in } 10 \mathrm{~g} \\
\text { Leberbrei } \\
\text { in } \mathrm{g}\end{array}$ & $\begin{array}{l}\text { Nichtkoagu- } \\
\text { labler Stick- } \\
\text { stoff in } 10 \mathrm{~g} \\
\text { Leberbrei } \\
\text { in } \mathrm{g}\end{array}$ & $\begin{array}{c}\text { Prozent- } \\
\text { gehalt des } \\
\text { Gesamtstick- } \\
\text { stoffs }\end{array}$ & $\begin{array}{l}\text { Prozent- } \\
\text { gehalt des } \\
\text { nichtkoagu- } \\
\text { lablen Stick- } \\
\text { stoffs }\end{array}$ & $\begin{array}{l}\text { Prozentver- } \\
\text { hältnis des } \\
\text { nichtkoagu- } \\
\text { lablen zu dem } \\
\text { Gesamtstick- } \\
\text { stoff }\end{array}$ \\
\hline 1. & 0,35496 & 0,02275 & 3,55 & 0,23 & 6,41 \\
\hline 2. & 0,36882 & 0,03105 & 3,69 & 0,31 & 8,42 \\
\hline 3. & 0,25657 & 0,01859 & 2,57 & 0,19 & 7,24 \\
\hline 4. & 0,37110 & 0,03094 & 3,71 & 0,31 & 8,34 \\
\hline 5. & 0,34655 & 0,02500 & 3,47 & 0,25 & 7,21 \\
\hline 6. & 0,37696 & 0,03577 & 3,77 & 0,36 & 9,48 \\
\hline 7. & 0,34110 & 0,02797 & 3,41 & 0,28 & 8,20 \\
\hline 8. & 0,35871 & 0,02995 & 3,59 & 0,30 & 8,34 \\
\hline
\end{tabular}


schweinchenleber etwa $10 \mathrm{~g}$ beträgt, sind der besseren Übersicht wegen die erbaltenen Stickstoffwerte auf $10 \mathrm{~g}$ Feuchtgewicht des zur Analyse verwendeten Organbreies berechnet.

Es enthält somit die normale Meerschweinchenleber im Durchschnitt etwa $3,47 \%$ an Gesamtstickstoff und $0,28 \%$ an Stickstoff nicht koagulierbarer Körper, bezogen auf das Gewicht des frischen Organbreies, so daß unter der gegebenen Fïtterung (Haferfütterung mit Kohlblättern) und dem entsprechenden Gewicht der Tiere der unkoagulierbare Stickstoff etwa $8 \%$ des Gesamtstickstoffs in der normalen Meerschweinchenleber beträgt. Wie man aus den Zahlen der letzten Kolumne der angeführten Versuchsreibe ersieht, schwankt das Prozentverhältnis des unkoagulablen Stickstoffs zum Gesamtstickstoff bei der Leber der untersuchten acht Tiere zwisehen 6,41 als niedrigsten und $9,48 \%$ als höchsten Wert. Es ist selbstverständlich, daß diese Zahlen nur für die Meerschweinchenleber gelten, und daß für andere Tierarten sich wieder andere Werte ermitteln lassen; so schwankt z. B. nach Schlesinger ${ }^{1}$ ) die Menge des nichtkoagulablen Leberstickstoffs bei normalen erwachsenen Kaninchen im Durchschnitt zwischen 15-20\% und soll bei neugeborenen Tieren sogar $60 \%$ des Gesamtstickstoffs erreichen; wir selbst trafen bei der Untersuchung von Lebern normaler Mäuse ebenfalls $17,5-18 \%$ des Gesamtstickstoffs an unkoagulablem Stickstoff an.

II. Über das Verhältnis des unkoagulablen Stickstoffs zu dem Gesamtstickstoff in der Leber von mit Pferdeserumeiweiß sensibilisierten Meerschweinchen.

Die Untersuchungen der Lebern dieser Versuchsreihe wurden in der gleichen Weise wie die vorhergehenden ausgeführt; alle Meerschweinchen wurden mit $1 / 2 \mathrm{ccm}$ nativem Pferdeserum subkutan vorbehandelt und nach 14-20 Tagen durch Entbluten getötet. Die Resultate der Leberanalysen von acht Meerschweinchen sind in folgender Tabelle zusammengestellt: 
Über d. intravitalen Eiweißabban in d. Leber sensibilisierter Tiere usw.

Tabelle 2.

\begin{tabular}{|c|c|c|c|c|c|c|}
\hline 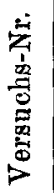 & $\begin{array}{c}\text { Anzahl der } \\
\text { seit der Vor- } \\
\text { behandlung } \\
\text { verflossenen } \\
\text { Tage }\end{array}$ & $\begin{array}{c}\text { Gesamt-N in } \\
10 \mathrm{~g} \text { Leber- } \\
\text { brei in } \mathrm{g}\end{array}$ & $\begin{array}{c}\text { Nichtkoagu- } \\
\text { labler } \mathrm{N} \text { in } \\
10 \mathrm{~g} \text { Leber- } \\
\text { brei in } \mathrm{g}\end{array}$ & 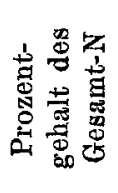 & 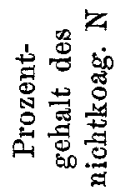 & $\begin{array}{l}\text { Prozentver- } \\
\text { hältnis des } \\
\text { nichtkoag. } \\
\text { zu dem Ge- } \\
\text { samt-N }\end{array}$ \\
\hline 1. & 14 & 0,38807 & 0,09269 & 3,88 & 0,93 & 23,89 \\
\hline 2. & 14 & 0,37608 & 0,08591 & 3,76 & 0,86 & 22,87 \\
\hline 3. & 15 & 0,40146 & 0,07912 & 4,01 & 0,79 & 19,71 \\
\hline 4. & 16 & 0,28587 & 0,06770 & 2,86 & 0,68 & 23,68 \\
\hline 5. & 18 & 0,30857 & 0,06263 & 3,09 & 0,63 & 20,29 \\
\hline 6. & 20 & 0,37141 & 0,07519 & 3,71 & 0,75 & 20,09 \\
\hline 7. & 14 & 0,37107 & 0,09003 & 3,71 & 0,90 & 24,25 \\
\hline 8. & 15 & 0,36578 & 0,07715 & 3,66 & 0,77 & 21,09 \\
\hline
\end{tabular}

Es ergibt sich bei Übersicht dieser Tabelle, daß der Gesamtstickstoff des Leberbreies der sensibilisierten Tiere gegenüber unvorbehandelten Meerschweinchen keinerlei Anderung erfahren hat, daB aber der Gehalt der sensibilisierten Meerschweinchenleber an Stickstoff nichthitzekoagulabler Körper in allen Versuchen beinahe auf das Dreifache des Gehaltes normaler Meerschweinchenlebern gestiegen ist; während bei den letzteren nur etwa $8 \%$ des Gesamt-N dem unkoagulablen Stickstoffanteil angehören, steigt dieses Prozentverhältnis in der vorbehandelten Leber auf etwa $22 \%$ im Mittel, wobei der niedrigste Wert 19,71 , der höchste $24,25 \%$ beträgt. Der besseren Übersicht wegen seien die Durchschnittswerte beider Versuchsreihen in nachfolgender Tabelle zusammengestellt.

Tabelle 3 .

Durchschnittswerte der Leberanalysen normaler und sensibilisierter Meersehweinchen.

\begin{tabular}{|c|c|c|c|c|c|c|c|}
\hline & 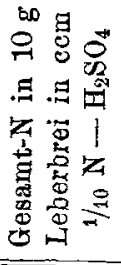 & 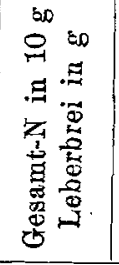 & 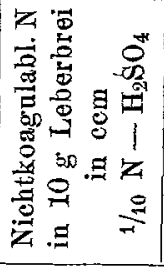 & 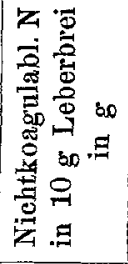 & 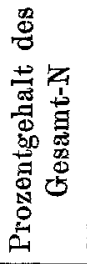 & 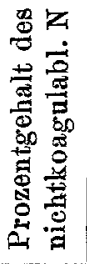 & 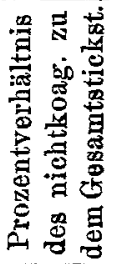 \\
\hline $\begin{array}{l}\text { unvorbehandelte } \\
\text { Meerschwein- } \\
\text { chen :. . } \\
\text { sensibilisierte } \\
\text { Meerschwein- }\end{array}$ & 247,75 & 0,34686 & 19,82 & 0,02776 & 3,47 & 0,28 & 8,08 \\
\hline$n \cdot$ & 256,09 & 0,35853 & $\tilde{5} 6,28$ & 0,07880 & 3,58 & 0,79 & 21,98 \\
\hline
\end{tabular}


Bei uverändertem Gesamtstickstoff zeigt demnach die Leber sensibisierter Meerschweinchen eine augenfällige Anreicherung an stickstoffhaltigen Stoffen, die den genuinen Eiweißkörpern nicht angehören, sondern wahrscheinlich Eiweißspaltprodukte darstellen, deren nähere Charakterisierung später erfolgen soll.

Diese Vermehrung stickstoffhaltiger Spaltprodukte in der Leber eiweißvorbehandelter, frisch getöteter Tiere würde von vornherein mit der Annahme jener Autoren gut übereinstimmen, welche den Abbau des eingeführten artfremden Eiweißes bei der Immunisierung voraussetzen und denselben mit dem Auftreten der Überempfindlichkeit in ursächlichen Zusammenhang bringen. Indessen zeigt schon die Berechnung der eingeführten Eiweißmengen und ein Vergleich derselben mit der gefundenen Zunahme an nicht koagulablem Leberstickstoff, daß die letztere unmöglich auf den Abban des zur Vorbehandlung verwandten artfremden Eiweißes zurïckgeführt werden kanu. Berechnet man den Eiweißgehalt des Pferdeserums zu $8 \%$, so entbält $0,5 \mathrm{ccm}$ desselben $0,04 \mathrm{~g}$ Eiweiß, entsprechend einer Stickstoffmenge von $0,0064 \mathrm{~g}$; selbst unter der sicher unzutreffenden Annahme, daß diese gesamte, subkutan injizierte Eiweißmenge in Form von Eiweißspaltprodukten in der Leber angehäuft würde, könnte sie nur eine kaum nennenswerte Vermehrung von etwa $2 \%$ an unkoagulablem Leberstickstoff bedingen. Dazu kommat noch, daB es uns gelang, eine ebenso bedeutende Zunahme stickstoffhaltiger Spaltprodukte in der Leber auch bei jenen Tieren nachzuweisen, deren Vorbehandlung mit einer einmaligen subkatanen Injektion von 0,001 ccm Pferdeserum, entsprechend einer Eiweißmenge von $0,00008 \mathrm{~g}$ und einer Stickstoffmenge von $0,0000128 \mathrm{~g}$, durehgefuhrt worden war. Die entsprechenden Daten gehen aus nachfolgender Tabelle hervor.

Tabelle 4.

\begin{tabular}{|c|c|c|c|c|c|c|}
\hline $\begin{array}{c}\text { Versuch } \\
\mathrm{Nr}^{*}\end{array}$ & $\begin{array}{c}\text { Anzahl } \\
\text { der seitd. } \\
\text { Vorbe- } \\
\text { handlung } \\
\text { verfloss. } \\
\text { Tage }\end{array}$ & $\begin{array}{l}\text { Gesamt-N } \\
\text { in } 10 \mathrm{~g} \\
\text { Leberbrei } \\
\mathrm{g}\end{array}$ & $\begin{array}{l}\text { Nichtkoag. } \\
\mathrm{N} \text { in } 10 \mathrm{~g} \\
\text { Leberbrei } \\
\mathrm{g}\end{array}$ & $\begin{array}{c}\text { Gehalt } \\
\text { des Ge- } \\
\text { samt-N } \\
0 \%\end{array}$ & $\begin{array}{c}\text { Gehalt } \\
\text { des nicht- } \\
\text { koag. } \mathbf{N} \\
\%\end{array}$ & $\begin{array}{c}\text { Verhältnis } \\
\text { des nicht- } \\
\text { koag. zu d. } \\
\text { Gesamt-N } \\
0 / 0 \\
\end{array}$ \\
\hline $\begin{array}{l}1 \\
2 \\
3 \\
4 \\
5\end{array}$ & $\begin{array}{l}14 \\
15 \\
18 \\
21 \\
21\end{array}$ & $\begin{array}{l}0,28455 \\
0,26624 \\
0,34987 \\
0,29878 \\
0,26171\end{array}$ & $\begin{array}{l}0,04640 \\
0,04263 \\
0,06858 \\
0,06202 \\
0,06254\end{array}$ & $\begin{array}{l}2,85 \\
2,66 \\
3,50 \\
2,99 \\
2,62\end{array}$ & $\begin{array}{l}0,46 \\
0,43 \\
0,69 \\
0,62 \\
0,63\end{array}$ & $\begin{array}{l}16,31 \\
16,01 \\
19,60 \\
20,75 \\
23,89\end{array}$ \\
\hline In Mittel & $14-21$ & 0,29223 & 0,05643 & 2,92 & 0,57 & 19,31 \\
\hline
\end{tabular}


Es unterliegt daher keinem $\mathrm{Z}$ weifel, daß die vorgefundenen Eiweißspaltprodukte in der Leber eiweiß-sensibilisierter Tiere nicht von dem zur Vorbehandlung benütaten artfremden Eiweiß abstammen, sondern nurdurch Zerfall des arteigenen Eiweißes unter der Einwirkung der Eiweißvorbehandlung entstanden sein können. Die letzte Versuchsreihe zeigt außerdem, daß schon eine einmalige Injektion von Hundertsteln eines Milligrammes artfremden Eiweißes genügt, um diesen höchst merkwürdigen Abbau des Körpereiweißes qualitativ und quantitativ in derselben Weise herbeizuführen, wie die in den früheren Versuchsreihen beniitzte 500 fach größere Eiweißmenge; bedenkt man, daß die Sensibilisierung noch mit weit geringeren Eiweißmengen, als sie hier verwendet worden sind, gelingt, so erbält man eine Vorstellung dariiber, wie hoch empfindlich der tierische Eiweißstoffwechsel gegenüber Eingriffen dieser Art ist. Da nicht allein körperfremdes Eiweiß, sondern, wie zahlreiche Erfahrungen bei Verwendung ron körpereigenen Organextrakten lehren, schon körpereigene, jedoch blutfremde Eiweißkörper zu sensibilisieren vermögen, ist es naheliegend, analoge Eiweißzerfallsprozesse anch bei denjenigen teils physiologischen (Schwangerschaft), teils pathologischen Zuständen zu vermuten, bei denen blutfremdes Eiweißmaterial in Zirkulation gesetzt wird; da schon die geringsten Mengen tiefgreifende Veränderungen im normalen Eiweißabban setzen, erscheint es leicht begreiflich, daß derartige Prozesse schon fribzeitig eine gewaltige Umstimmung des normalen Eiweißstoffwechsels herbeiführen können, und es wird Aufgabe weiterer Untersuchungen sein, festzustellen, ob nicht bei derartigen Prozessen, wie bei der Schwangerschaft, dem Tumorwachstum (Krebskachexie), manchen Vergiftungen (Phosphor, Arsen) und Infektionen analoge Veränderungen im Organeiweißhaushalte vorliegen, wie sie bei der Eiweißsensibilisierung künstlich erzengt werden können.

Wie später noch gezeigt werden soll, ist von allen Organen hauptsächlich die Leber an der Bildung der bei der Eiweißsensibilisierung auftretenden Eiweißspaltprodukte beteiligt; es wird daher von besonderem Interesse sein, speziell die mit dem Eiweißabbau in der Leber einhergehenden pathologischen Prozesse, wie die akute gelbe Leberatrophie und akute Phosphorvergiftung auf ihre Beziehung za den eben erörterten Erscheinungen zu prüfen. 
III. Über das Verhalten des sunkoagulablen * Stickstoffes in Nieren, Milz, Gehirn und Blut normaler und sensibilisierter Meerschweinchen.

Die ansehnliche Vermehrung der unkoagulablen Eiweißkörper in der Leber sensibilisierter Tiere regte $\mathrm{zu}$ gleichartigen Untersuchungen anderer Organe vorbehandelter Meerschweinchen an, um zunächst zu entscheiden, ob der in der Leber vorhandene Eiweißzerfall anch die anderen Organe betrifft oder der Leber allein oder vorzugsweise eigentümlich ist. $\mathrm{Zu}$ diesem Behufe wurden in derselben Weise, wie bei der Leber die Nieren, die Milz and das Gehirn sowohl normaler als auch mit Pferdeserum vorbehandelter Meerschweinchen untersucht; die Untersuchung des Gehirns wurde deshalb durchgeführt, weil nach Abelous und Bardier') im Gefolge einer Antigeninjektion Änderungen im Zentralnervensystem eintreten sollen, die sich nach neuesten Angaben von Soula, bei Verwendung von Ovalbumin und Urohypotensin als Antigen, in einer »Autoproteolyse der nervösen Zentren " äußern. Besondere Aufmerksamkeit wurde auch der Blutuntersuchnng zugewandt, weil einige, einander allerdings widersprechende Angaben über das Auftreten von Biuretreaktion gebenden Eiweißspaltprodukten im Blute anaphylaktischer Tiere vorliegen und die Möglichkeit des Erscheinens derartiger Produkte im Blute schon während der Sensibilisierung gegeben war. Die Untersuchung des Blutes fand derart statt, daß die Tiere aus der Carotis entblutet worden sind und das sofort defibrinierte Blut in gleicher Weise, wie die Organe der Stickstoffbestimmung und Koagulation unterzogen worden sind; für je eine Bestimmang wurden stets $4 \mathrm{ccm}$ des defibrinierten Blutes verwendet. Die genaneren Versuchsdaten sowie die Analysenergebnisse sind aus den folgenden tabellarischen Zusammenstellungen ersichtlich.

Die Analysen der untersuchten Organe zeigen übereinstimmend, daß in keinem derselben nach der Sensibilisierung der Meerschwein. schen mit Pferdeserum eine nennenswerte Änderung im Gehalte der unkoagulablen Eiweißkörper gegenüber der Norm eingetreten war und daß auch die Verteilung der hitzekoagulablen und ungerinnbaren Proteine im Blute der vorbehandelten und normalen Tiere dieselbe ist; insbesondere aber haben sich für eine, übrigens von vornherein wenig

1) Abelous, J. F. und Bardier, Compt. rend. d. l'Académ. des sciences T. 154, p. 1529; Soula, L. C., ebenda T. 156, p. 1258. 
Tabelle 5 .

Untersuchungen der Nieren bei normalen und sensibilisierten Meerschweinchen.

\begin{tabular}{|c|c|c|c|c|c|c|}
\hline $\begin{array}{l}\text { Versuchs- } \\
\text { nummer }\end{array}$ & $\begin{array}{l}\text { Gesamt- } \\
\text { stickstoff } \\
\text { in } 2,5 \mathrm{~g} \\
\text { Nierenbrei } \\
\mathrm{g} \\
\end{array}$ & $\begin{array}{l}\text { Unkoagul. } \\
\text { Stickstoff } \\
\text { in } 2,5 \mathrm{~g} \\
\text { Nierenbrei } \\
\mathrm{g} \\
\end{array}$ & 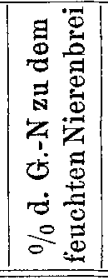 & 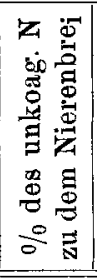 & $\begin{array}{c}\% \% \text { des } \\
\text { unkoagul. } \\
\text { Stick- } \\
\text { stoffs z. } \\
\text { Gesamt- } \\
\text { stickstoff }\end{array}$ & $\begin{array}{l}\text { Tage } \\
\text { nach } \\
\text { der } \\
\text { Injek- } \\
\text { tion }\end{array}$ \\
\hline $\begin{array}{c}\text { Norm. Nr. } 1 \\
>\quad \text { Nr. } 2 \\
>\quad \text { Nr. } 3 \\
\Rightarrow \quad \text { Nr. } 4 \\
\text { Durchschnittl. } \\
\text { Wert }\end{array}$ & $\begin{array}{c}0,06346 \\
0,06990 \\
0,07058 \\
0,06783 \\
48,53 \mathrm{ccm} \frac{\mathrm{N}}{10} \\
\mathrm{H}_{2} \mathrm{SO}_{4}=0,06794\end{array}$ & $\begin{array}{c}0,01232 \\
0,01400 \\
0,01336 \\
0,01621 \\
9,98 \mathrm{~cm} \frac{\mathrm{N}}{10} \\
\mathrm{H}_{2} \mathrm{SO}_{4}=0,01397\end{array}$ & $\begin{array}{l}2,54 \\
2,80 \\
2,82 \\
2,82 \\
2,75 \%\end{array}$ & $\begin{array}{l}0,49 \\
0,56 \\
0,53 \\
0,65\end{array}$ & $\begin{array}{l}19,41 \\
20,29 \\
18,93 \\
23,90 \\
20,63 \%\end{array}$ & $\begin{array}{l}- \\
- \\
-\end{array}$ \\
\hline \begin{tabular}{cr} 
Sengibil. & Nr. 1 \\
$»$ & Nr. 2 \\
$»$ & Nr. 3 \\
$»$ & Nr. 4 \\
\multicolumn{3}{c}{ Durchschnittl. } \\
\multicolumn{2}{c}{ Wert }
\end{tabular} & $\begin{array}{c}0,06790 \\
0,09361 \\
0,07469 \\
0,06748 \\
54,22 \mathrm{ccm} \frac{\mathrm{N}}{\mathbf{1 0}} \\
\mathrm{H}_{2} \mathrm{SO}_{4}=0,07592\end{array}$ & $\begin{array}{c}0,01686 \\
0,02353 \\
0,01551 \\
0,01726 \\
13,06 \mathrm{~cm} \frac{\mathrm{N}}{\mathbf{1 0}} \\
\mathrm{B}_{2} \mathrm{SO}_{4}=0,01829\end{array}$ & $\begin{array}{l}2,72 \\
3,74 \\
2,99 \\
2,70 \\
3,04 \%\end{array}$ & $\begin{array}{l}0,67 \\
0,94 \\
0,62 \\
0,70\end{array}$ & $\begin{array}{r}24,83 \\
25,14 \\
20,77 \\
25,57 \\
24,08 \%\end{array}$ & $\begin{array}{l}14 \\
15 \\
18 \\
15\end{array}$ \\
\hline
\end{tabular}

Tabelle 6.

Untersuchungen der Milzen bei normalen und sensibilisierten Meerschweinchen.

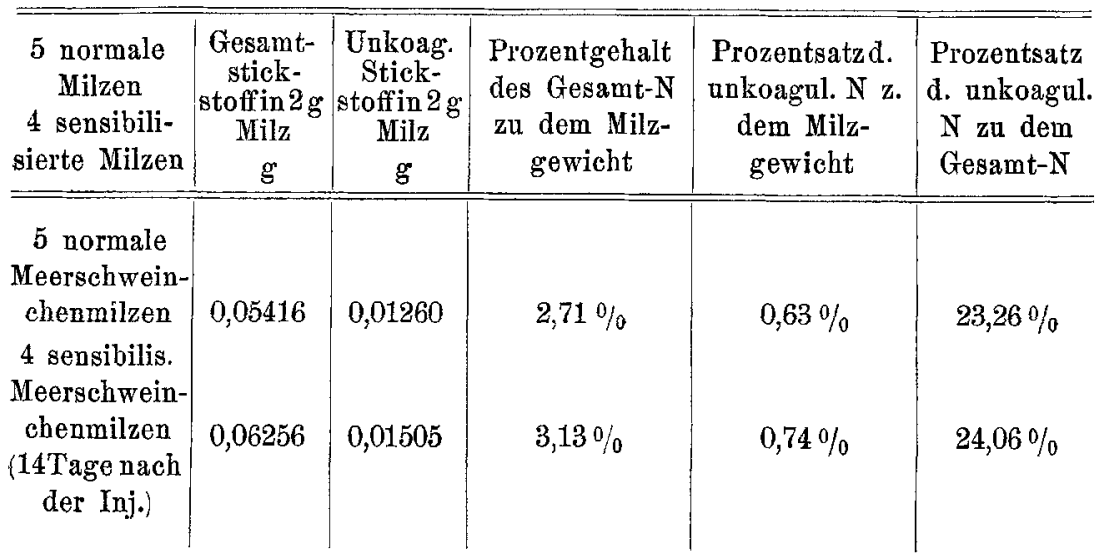


Tabelle 7 .

Untersuchungen des Gehirns normaler und sensibilisierter Meerschweinchen.

\begin{tabular}{|c|c|c|c|c|c|c|}
\hline $\begin{array}{l}\text { Versuchs- } \\
\text { nummer }\end{array}$ & $\begin{array}{l}\text { Gesamt- } \\
\text { stickstoff } \\
\text { in } 3 \mathrm{~g} \\
\text { Gehirn } \\
\mathrm{g}\end{array}$ & $\begin{array}{c}\text { Unkoagul.- } \mathrm{N} \\
\text { in } 3 \mathrm{~g} \\
\text { Gehirn } \\
\mathrm{g} \\
\end{array}$ & 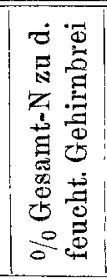 & 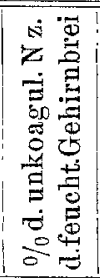 & $\begin{array}{c}\% \text { des } \\
\text { unkoagul. } \\
\text { N z. d. } \\
\text { Gesamt- } \\
\text { N }\end{array}$ & $\begin{array}{l}\text { Tage } \\
\text { nach } \\
\text { der } \\
\text { Injek- } \\
\text { tion }\end{array}$ \\
\hline Norm. Nr. 1 & 0,05103 & 0.01002 & 1,70 & 0,33 & 19 & - \\
\hline$\Rightarrow \mathrm{Nr}$. & & & 1,7 & 036 & & - \\
\hline$\gg \quad \mathrm{Nr} .3$ & 0,05118 & 0,00911 & 1,71 & 0,30 & 17,80 & - \\
\hline Durchsehnitt & $\underset{\mathrm{H}_{2} \mathrm{SO}_{4}}{36,64 \mathrm{ccm}} \frac{\mathrm{N}}{10}$ & $\underset{\mathrm{H}_{2} \mathrm{SO}_{4}}{7,11 \mathrm{ccm}} \frac{\mathrm{N}}{10}$ & $1,71 \%$ & $|0,33 \%|$ & $19,38 \%$ & - \\
\hline nsibil. Nr. & 0,059 & 0,008 & $\overline{1,9}$ & 0,32 & $\overline{1 k}$ & 14 \\
\hline $\mathrm{Nr}$, & & & 1,9 & 0,24 & & 18 \\
\hline Nr. 3 & 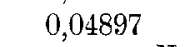 & & 1,63 & 0,30 & 18,15 & 20 \\
\hline Durchschnitt & $\underset{\mathrm{H}_{2} \mathrm{OS}_{4}}{43,19 \mathrm{ccm}} \frac{\mathrm{N}}{10}$ & $\begin{array}{c}6,12 \mathrm{ccm} \\
\mathrm{H}_{2} \mathrm{SO}_{4} \\
\frac{\mathrm{N}}{10}\end{array}$ & $1,86 \%$ & $0,29 \%$ & $15,46 \%$ & - \\
\hline
\end{tabular}

Tabelle 8.

Untersuchungen des Blutes bei normalen und sensibilisierten Meerschweinchen.

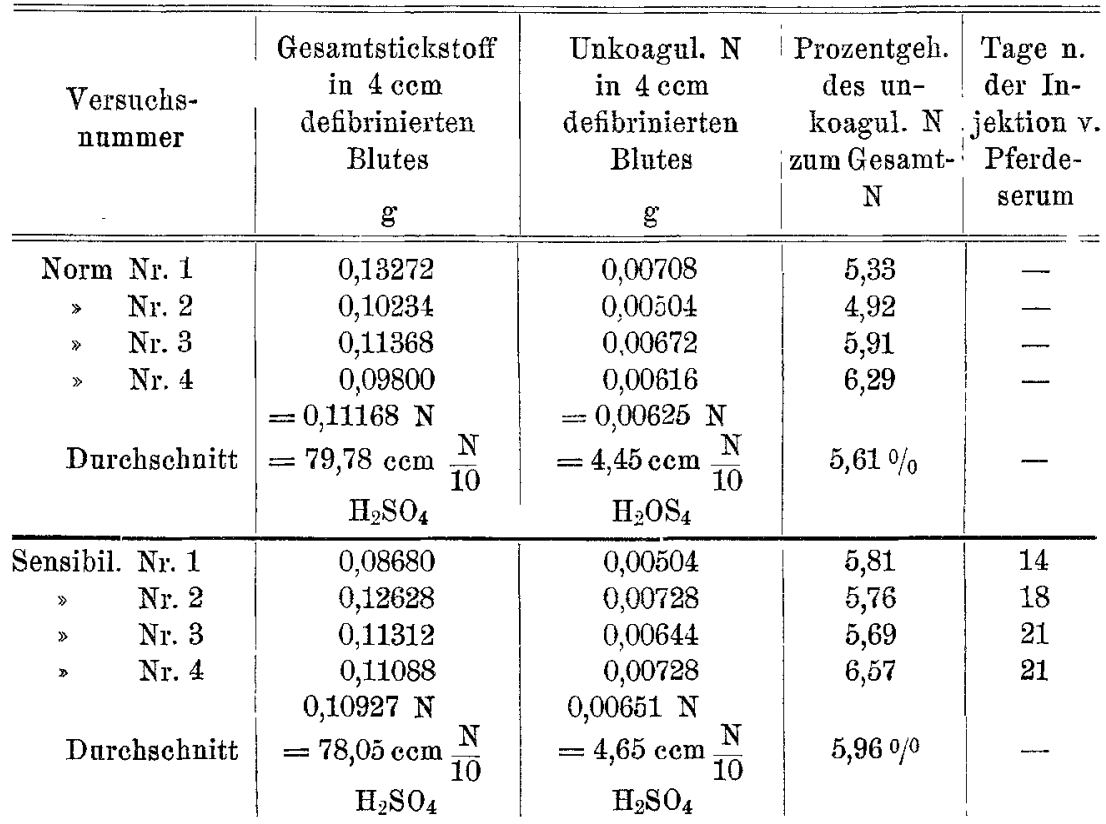


Über d. intravitalen Eiweißabbau in d. Leber sensibilisierter Tiere usw. 101

wahrscheinliche, Steigerung der Proteolyse im Zentralnervensystem der sensibilisierten Meerschweinchen keinerlei Anhaltspunkte ergeben. Die in den untersuchten Organen und im Blute gefundenen geringen Differenzen bei den vorbehandelten und unvorbehandelten Tieren entsprechen individuellen Schwankungen and können in keiner Weise mit den großen, bei der Leber erhaitenen Unterschieden verglichen werden. Bemerkenswert ist das negative Ergebnis bei der Milz, der man, wie den lymphoiden Organen iberhaupt, ursprïnglich bei der Antikörperbildung eine Rolle zugeschrieben batte; nach den hier vorliegenden Befunden beteiligt sich die Milz in direkter Weise jedesfalls nicht an dem durch die Eiweißsensibilisierung angeregten Eiweißabbau. In jîngster Zeit haben Auer and van Slyke ${ }^{1}$ ) auch Herz und Lunge von im anaphylaktischen Shock zugrunde gegangenen Meerschweinchen in bezug aut das Vorhandensein von Eiweißspaltungsprodukten mit negativem Resultat untersucht. Sieht man ab von der hier nicht näher untersuchten quergestreiften Muskulatur und einigen Organen (Sehilddriise, Nebennieren, Hypophyse), für deren direkte Beteiligung an den hier in Frage stehenden Beobachtungen kein Grund vorliegt, so ergibt sich ans den angeführten Befunden, daß die Leber ausschließlich oder wenigstens in herrorragendstem Maße den durch die Eiweißsensibilisierung hervorgerufenen Abbau des Körpereiweißes besorgt.

IV. Über den zeitlichen Zusammenhang zwischen Sensibilisierung und dem Auftreten intravitaler Leberautolyse.

In den schon eingangs erwähnten Arbeiten von Friedemann und Isaak, insbesondere aber von Heilner wurde festgestellt, daß das auf subkutanem Wege eingefuhrte artfremde Eiweiß in den nächsten der Injektion folgenden 3 Tagen eine vermehrte Stickstoffausscheidung bedingt, aus der die relativ rasche Verbrennung der mit Umgehung des Darmkanals in die Gewebssäfțe gelangten Eiweißes erschlossen wurde. Es war von Interesse, festzustellen, ob auch der von uns beobachtete Lebereiweißabbau im unmittelbaren Anschluß an die EiweiBinjektion einsetzt oder erst später, mit dem Beginn der Sensibilisierung und Immunkörperbildung, in Erscheinung tritt. Zu diesem Behufe warden Meerschweinchen in der früher geschilderten Weise mit Pferdeserum vorbehandelt, in verschiedenen Zeiten nach der sensibilisierenden Injektion getötet und die Leber in der iiblichen Weise verarbeitet; auf diese Weise wurden in fortlaufender Reihe

1) Auer und van Slyke, Zentralblatt f. Physiologie Bd. 27, 1913, S. 435. 
Tiere mit 3-68tägigem Intervall nach der Eiweißinjektion untersucht. Die Resultate, die aus der nachfolgenden Tabelle 9 zu er-

Tabelle 9 .

\begin{tabular}{|c|c|c|c|c|c|c|}
\hline 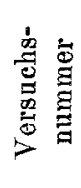 & $\begin{array}{l}\text { Gesamt- } \\
\text { stickstoff in } \\
10 \mathrm{~g} \text { Leber } \\
\mathrm{g}\end{array}$ & $\begin{array}{l}\% \text { des Ge- } \\
\text { samt-N zum } \\
\text { Leber- } \\
\text { gewicht }\end{array}$ & $\begin{array}{c}\text { unkoagulab- } \\
\text { ler N in } \\
10 \mathrm{~g} \text { Leber } \\
\mathrm{g}\end{array}$ & $\begin{array}{c}\% \text { des un- } \\
\text { koagulabl. } \mathrm{N} \\
\text { zam Leber- } \\
\text { gewicht }\end{array}$ & $\begin{array}{c}0 / 0 \text { des un- } \\
\text { koagulablen } \\
\mathrm{N} \text { zum Ge- } \\
\text { samt-N }\end{array}$ & 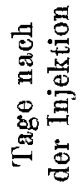 \\
\hline 1 & 0,37239 & 3,72 & 0,03643 & 0,36 & 9,78 & 3 \\
\hline 2 & 0,37715 & 3,77 & 0,03812 & 0,38 & 10,11 & 4 \\
\hline 3 & 0,44154 & 4,42 & 0,04074 & 0,41 & 9,22 & 5 \\
\hline 4 & 0,31726 & 3,17 & 0,03630 & 0,36 & 11,44 & 6 \\
\hline$\tilde{\sigma}$ & 0,37841 & 3,78 & 0,04573 & 0,46 & 12,09 & 7 \\
\hline 6 & 0,35980 & 3,60 & 0,04301 & 0,43 & 11,95 & 7 \\
\hline 7 & 0,29878 & 2,99 & 0,04565 & 0,46 & 15,28 & 8 \\
\hline 8 & 0,26171 & 2,62 & 0,04240 & 0,42 & 16,14 & 8 \\
\hline 9 & 0,27922 & 2,79 & 0,04258 & 0,43 & 15,25 & 9 \\
\hline 10 & 0,34489 & 3,45 & 0,06321 & 0,63 & 18,33 & 10 \\
\hline 11. & 0,31725 & 3,17 & 0,05682 & 0,57 & 17,91 & 11 \\
\hline 12 & 0,33252 & 3,32 & 0,06348 & 0,64 & 19,09 & 12 \\
\hline 13 & 0,24568 & 2,46 & 0,05417 & 0,54 & 22,04 & 13 \\
\hline 14 & 0,37107 & 3,71 & 0,09000 & 0,90 & 24,25 & 14 \\
\hline 15 & 0,36578 & 3,66 & 0,07714 & 0,77 & 21,09 & 16 \\
\hline 16 & 0,30856 & 3,09 & 0,06263 & 0,63 & 20,30 & 18 \\
\hline 17 & 0,37141 & 3,71 & 0,07519 & 0,75 & 20,24 & 20 \\
\hline 18 & 0,34443 & 3,44 & 0,07292 & 0,73 & 21,17 & 21 \\
\hline 19 & 0,34987 & 3,50 & 0,06859 & 0,69 & 19,61 & 24 \\
\hline 20 & 0,30724 & 3,07 & $0,05 \tilde{6} 6 \tilde{8}$ & 0,57 & 18,42 & 28 \\
\hline 21 & 0,31052 & 3,11 & $0,0 ذ 049$ & 0,50 & 16,26 & 38 \\
\hline 22 & 0,28338 & 2,83 & 0,04468 & 0,45 & 15,77 & 48 \\
\hline 23 & 0,29948 & 2,99 & 0,03928 & 0,39 & 13,12 & 58 \\
\hline 24 & 0,33538 & 3,35 & 0,04640 & 0,46 & 13,83 & 68 \\
\hline
\end{tabular}

sehen sind, zeigen, daß vom 3,-5. Tage angefangen in einer allmählich bis zum 14. Tage ansteigenden Kurve (siehe Fig. 1) die Menge des unkoagulablen Stickstoffes in der Leber zunimmt, um dann langsam abzunehmen; die Vermehrung der stickstoffhaltigen Abbanprodukte ist jedoch selbst 68 Tage nach der Eiweißinjektion sehr deutlich nachweisbar und beträgt $13,83 \%$ gegenüber der normalen Durchschnittszahl von etwa $8 \%$ des Gesamtstickstoffs an unkoagulablen Abbauprodukten. Man ersieht zunächst aus dem Verlaufe dieser Eiweißabbaukurve auf das dentlichste, daß der hier in Frage kommende LebereiweiBabbau durchaus unabhängig von der 
Über d. intravitalen Eiweißabban in d. Leber sensibilisierter Tiere usw. 103

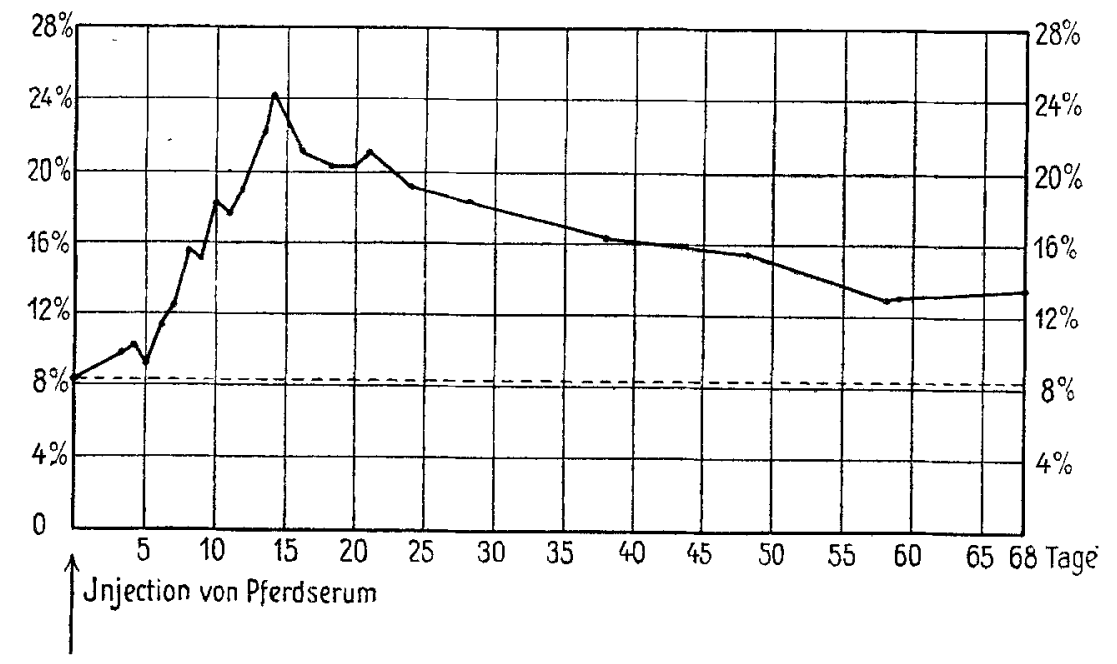

Figur 1:

Ordinate bezeichnet den Prozentsatz der unkoagulablen stickstoffhaltigen Stoffe vom Gesamtstickstoff, die Abszisse die Anzahl der seit der sensibilisierenden Seruminjektion verflossenen Tage.

Verbrennung des zugeführten, artfremden Eiweißes erfolgt, die nach den vorliegenden Erfahrungen bei der geringen, hier angewandten Menge zum größten Teil unmittelbar nach der Injektion bätte erfolgen müssen; es ist im Gegenteil zu beobachten, daß der Eiweißabbau erst etwa mit dem 3 . Tage beginnt and ron da ab stetig bis zum 14. und 16. Tage zunimmt, das Maximum daher zu einer Zeit erreicht, in der die geringfügige, subkutan einverleibte Eiweißmenge schon längst einer Zersetzung und Ausscheidung anheimgefallen wäre. Dagegen ist eine angenfällige Übereinstimmung zwischen der Eiweißabbau- und Sensibilisierungskurve wahrzunehmen; denn wir wissen sowohl aus den Untersuchnogen anderer ${ }^{1}$, als auch ans eigener Erfahrung, daß bei der von uns zur Vorbehandlang verwendeten Eiweißmenge die Empfindlichkeit der Tiere gegenüber der Reinjektion von Eiweiß ebenfalls allmählich zunimmt, gegen Ende der 2. oder mit Beginn der 3. Woche am stärksten wird und von da sehr langsam abnimmt. Doch auch nach 70 Tagen, zur Zeit also, wo der Lebereiweißabbau zwar im Ablslingen, jedoch noch immer gegen die Norm gosteigert ist, waren auch unsere Tiere, in Übereinstimmung mit den

1) Siehe die einschlägige Literatur bei R Doerr, Allergie und Anaphylaxie im Handbach der pathogenen Mikroorganismen von Kolle und Wassermann Bd. II, S. 947, 1913. 
Angaben anderer Autoren, derart überempfindlich, daß sie durch Reinjektion von $2 \mathrm{ccm}$ intravenös beigebrachtem Pferdeserum im anaphylaktischen Shock getötet werden konnten. Die große Übereinstimmung im zeitlichen Ablauf des intravitalen Leberabbaues und der Überempfindlichkeit der vorbehandelten Meersehweinchen legt einen Zusammenhang der beiden Erscheinungen nahe; trotzdem läBt sich vorläufig nur die gemeinsame Abhängigkeit beider Erscheinungen von der Einfuhr des artfremden Eiweißes sicherstellen, ohne daß eine direkte gegenseitige Abhängigkeit des Leberabbaues und der durch die Auslösung des tödlichen anaphylaktischen Bronchospasmus charakterisierten Überempfindlichkeit des Meerschweinchens nötig wäre; im Folgenden anzuführende Versuche machen es vielmehr wahrscheinlich, daß die gesteigerte intravitale Leberproteolyse und die auf erhöhte Erregbarkeit der Vagusendigungen zu beziehende anaphylaktische Lungenstarre voneinander unabhängige, nebeneinander verlaufende Reaktionen verschiedener Zellen sind, welche unter dem Einfluß eines gemeinsamen Agens (Immunkörpers?) je nach ihren spezifischen Qualitäten in ihrer Reaktionsfähigkeit geändert worden waren.

V. Über den Einfluß wiederholter Eiweißinjektion auf die intravitale Leberautolyse.

Bekanntiich tritt die Eiweißüberempfindlichkeit am deutlichsten nach einer einmaligen Eiweißinjektion hervor, während eine zweite, in einem bestimmten kurzen Intervall der ersten nachfolgende Eiweißinjektion nicht nur keine Steigerung, sondern vielmehr eine völlige Hemmung der Überempfindlichkeit, also Unempfindlichkeit Antianaphylaxie) des betreffenden Tieres gegen eine sonst tödliche Dosis des zur Vorbebandlung benitzten Eiweißes hervorrufen kann. Es war daher von Wichtigkeit, zu prüfen, wie sich der Leberabban von Tieren verhält, die wiederholt mit Eiweib vorbehandelt und auf diese Weise nicht mehr sensibilisiert, sondern immunisiert worden waren. $\mathrm{Zu}$ diesem $\mathrm{Zweck}$ wurden Meerschweinchen von demselben Gewicht, wie früher in Intervallen von 4 Tagen $4-5$ mal mit je 1 cem Pferdeserum subkutan injiziert und 4 Tage nach der letzten, also 20 bís 21 Tage nach der ersten Injektion, durch Entbluten ans der Carotis getötet. Die unmittelbar nach dem Tode in bezug auf die Stickstoffverteilung verarbeitete Leber lieferte aus folgender Tabelle ersichtliche Werte: 
Über $d$. intravitalen Eiweißabbau in $d$. Leber sensibilisierter Tiere usw. 105

Tabelle 10.

Untersuchung der Leber der wiederholt mit Pferdeserum vorbehandelten Meerschweinchen.

\begin{tabular}{|c|c|c|c|c|c|c|}
\hline $\begin{array}{c}\text { Vers.- } \\
\text { Nr. }\end{array}$ & $\begin{array}{c}\text { Gesamt- } \\
\mathrm{N} \text { in } 10 \mathrm{~g} \\
\text { Leber } \\
\mathrm{g}\end{array}$ & $\begin{array}{c}\text { Durch } \\
\text { Hitze nicht } \\
\text { koagul. N } \\
(10 \mathrm{~g} \text { Leber }) \\
\mathrm{g}\end{array}$ & $\mid \begin{array}{c}0 / 0 \text { des Ge- } \\
\text { samt-N vom } \\
\text { Leber- } \\
\text { gewicht } \\
\text { g }\end{array}$ & $\begin{array}{l}\text { 0/0 des un- } \\
\text { koagul. } \mathrm{N} \\
\text { vom Leber- } \\
\text { gewicht }\end{array}$ & $\begin{array}{l}\text { \%o des un- } \\
\text { koagul. } \mathrm{N} \\
\text { yom } \\
\text { Gesamt-N }\end{array}$ & $\begin{array}{l}\text { Wie oft } \\
\text { injiziert? } \\
\text { Jeden. } \\
\text { 4. Tag }\end{array}$ \\
\hline 1$)$ & 0,29286 & 0,04042 & 2,93 & 0,40 & 13,84 & 4 \\
\hline 2 & 0,30993 & 0,04888 & 3,10 & 0,49 & 15,77 & 4 \\
\hline 3$)$ & 0,31630 & 0,04931 & 3,16 & 0,49 & 15,59 & 4 \\
\hline 4 & 0,29131 & 0,03168 & 2,91 & 0,32 & 10,87 & 5 \\
\hline 5 & 0,27303 & 0,03854 & 2,73 & 0,39 & 14,12 & 5 \\
\hline 6 & 0,28700 & 0,035552 & 2,87 & 0,36 & 12,38 & 5 \\
\hline
\end{tabular}

Es ergibt sich, daß kein einziges der Tiere jenen hochgradigen Abbau des Lebereiweißes aufweist, wie wir es bei den einmal mit Eiweiß vorbehandelten, sensibilisierten Tieren nach dem gleichen Intervall gesehen haben. Die 20 und 24 Tage nach der einmaligen Eiweißinjektion untersuchten Tiere zeigten, daß 20 bzw. 19\% des Leberstickstoffes unkoagulablen Produkten zugehört, während wir bei den sechs wiederholt gespritzten Tieren im Mittel nur einen Gehalt von 13,76\% an unkoagulablem Stickstoff finden. Es ist daher zweifellos bei den wiederholt mit Eiweiß vorbehandelten Meerschweinchen eine sehr deutliche Hemmung der sonst nach einmaliger EiweiBinjektion (Sensibilisierung) gesteigerten intravitalen Leberautolyse eingetreten, die in einem Falle (Versuchs-Nr. 4) sogar eine Zurückdrängung des Leberzerfalls bis nahezu zur Norm $(10,87 \%)$ bedingt hat. Dieses Verhalten bietet eine sehr sinnfällige Analogie zu dem antianaphylaktischen Zustand, wie er durch wiederholte Eiweißvorbehandlung innerhalb eines gewissen Zeitraumes erzielt werden kann. Ebenso wie hier die größere Menge der gebildeten Antikörper für das Ausbleiben des anaphylaktischen Zustandes wahrscheinlich verantwortlich ist, scheint sie auch der Entwicklung einer intensiveren Proteolyse der Leberzellen hinderlich zu sein.

VI. Über den Einfluß der Milz auf den intravitalen Leberabbau sensibilisierter Meerschweinchen.

Zahlreiche ältere und nevere Untersuchungen haben festgestellt, daß an der Immunkörperbildung die Milz und das übrige hämatopoe- 
tische System in hervorragendem Maße beteiligt sind. Andererseits sollten bereits nach alten Beobachtungen von Schiff ${ }^{1}$ ) in der Milz Stoffe vorhanden sein, welche proteolytische Zymogene zu aktivieren imstande wären, und durch neuere Arbeiten über das Wachstum experimentell erzeugter Tumoren wurde der wachstumhemmende und tumorenzerstörende Einfluß der Milz, der mit der Auslösung proteolytischer Zerfallsprozesse in den Zellen einhergeht, wiederholt sichergestellt (Borrel und Bridré, Braunstein, Oser und Přibram, Biach und Weltmann $)^{2}$. Endlich wurden sowohl auf experimentellem Wege (Joannorics ${ }^{3}$ ), Joannorics und Pick)i), als auch durch klinische Beobachtung (Eppinger) ${ }^{5}$ ) zahlreiche Beziehungen zwischen Milz und Leberstoffwechsel aufgedeckt. Alle diese Tatsachen legten es nahe, zu untersuchen, ob der Milz auch bei der Entstehung der intravitalen Leberautolyse eine Rolle zufällt.

Unsere Versuche führten wir derart aus, daß Meerschweinchen entweder 1-14 Tage vor der sensibilisierenden Pferdeseruminjektion oder auf der Höhe der Sensibilisierung, also 14 Tage nach der Injektion, die Milz exstirpiert worden war. Die vor der Sensibilisierung operierten Tiere wurden dann 14-15 Tage nach der sensibilisierenden Injektion, zur Zeit, wo normalerweise der Leberabbau am deutlichsten sich ausprägt, getötet, die erst auf der Sensibilisierungshöhe operierten Tiere blieben noch 6 Tage nach der Operation am Leben, gelangten daher zn einer Zeit zur Untersuchnng, in welcher nicht operierte, sensibilisierte Tiere ebenfalls einen sehr beträchtlichen Leberabban aufweisen. Als Kontrolle dienten unvorbehandelte Tiere, denen in gleicher Weise die Milz entfernt wurde. Die Milzexstirpation erfolgte unter aseptischen Kautelen in leichter Äthernarkose,

1) M. Schiff, Gesammelte Beiträge zur Physiologie 1868, Bd. 4, S. $143 \mathrm{ff}$.

2) Biach, P. und Weltmann, O., Über den wachstumhemmenden Einfluß der Milz auf das Rattensarkom. Wien. klin. Wochenschr. S. 1115, Jahrg. 1913, Nr. 27, daselbst auch die einschlägige Literatur.

3) Joannovics, G., Zeitschr. f. Heilkunde 1904, H. 1 und Recherches expér. sur la pathogénie de lictère. Mém. couron. publ. par l'acad. royal de méd. de Belg. Bruxelles 1903.

4) Joannovies und Pick, Beitrag zur Kenntnis der Toluylendiaminvergiftung. Zeitschr. f. exper. Path. u. Ther. 1009, Bd. 7, s. 185; Dieselben, Experimentelle Untersuchungen über die Bedentung der Leber bei der Fettresorption unter normalen und pathologischen Verbältnissen. Wiener klin. Wochenschr. 1910, Nr. 16.

5) H. Eppinger, Zur Pathologie der Milzfunktion. Berl. klio. Wochenschr. 1913, Nx. 33 u. 34. 
Über d. intravitalen Eiweißabbau in d. Leber sensibilisierter Tiere asw. 107

indem durch einen Schnitt in der Linea alba die Bauchhöhle eröffnet, das Milzmesenterium doppelt abgebunden und die Milz zwischen den Ligaturen rom Mesenterium mit einem Scherenschlag abgetrennt warde; hierauf warde die Bauchwand in Etagen vernäht. Es erfolgte stets Heilung per primam; bereits 1 Stunde nach der Operation zeigen die Tiere ein völlig normales Verhalten; sie wurden in gleicher Weise wie die anderen Tiere gefüttert. Die Sensibilisierung erfolgte auch bei diesen Tieren in der schon angeführten Weise. Der Tod der Tiere wurde in den meisten Fällen durch Verbluten aus der Carotis, in einigen, besonders bezeichneten, durch Frzeugung des anaphylaktischen Shockes nach intravenöser Injektion von 1-2 ccm Pferdeserum bewerkstelligt.

a) Sensibilisierungsversuche an entmilzten Tieren.

Die Resultate der einschlägigen Versuche lassen sich gut in der nachfolgenden Tabelle 12 überblicken. In den ersten fünf Versuchen, in denen die Sensibilisierung 1 Tag nach der Milzexstirpation vorgenommen worden war, zeigte die Leber der am 14.-16. Tage nach der sensibilisierenden Injektion getöteten Tiere ungefähr denselben Gehalt an koagulablem und unkoagulablem Stickstoff wie die Leber normaler, unvorbehandelter Meerschweinchen; das Mittel aus dem Prozentverhältnis des unkoagulablen Leber-N zum Gesamt-N beträgt 8,51\%. Diese Zahl stimmt gut ïberein mit der früher schon ermittelten der normalen, unvorbehandelten Meerschweinchen, die 8,08\% betrug, und deckt sich nahezu völlig mit den Zahlen, die sich aus einer Versuchsreihe ergaben, in welcher die Leber nicht sensibilisierter Meerschweinchen, denen die Milz 7-9 Tage vor dem Tode entfernt worden war, zur Untersuchung gelangte; das Mittel der bei diesen vier Tieren (Tabelle 11), die als Kontrolle dienen sollten, gefundenen Prozentzahlen betrug 8,72\% des unkoagulablen zum Gesamtstickstoff. Es verhalten sich demnach in bezug auf intravitalen Leberabbau normale, nicht sensibilisierte Tiere ebenso wie entmilzte Tiere, die unmittelbar nach der Milzexstirpation sensibilisiert worden waren.

In den weiteren vier Versuchen (Versuchs-Nr. 6-9) der Tabelle 12 wurde die Milz 15-17 Tage vor der sensibilisierenden Injektion exstirpiert, so daß der Tod wohl auf der Höhe der Sensibilisierung, aber erst etwa 30 Tage nach der Milzexstirpation erfolgte. Auch in diesen Versuchen sieht man, daß das Prozentverhältnis des unkoagulablen Stickstoffs zum Gesamtstickstoff bei weitem nicht die Höhe erreicht, wie wir sie bei den nichtentmilzten sensibilisierten Tieren 
Tabelle 11.

Untersuchung der Leber von entmilaten Meerschweinchen ohne Vorbehandlung mit Pferdeserum.

\begin{tabular}{|c|c|c|c|c|c|c|}
\hline $\begin{array}{l}\text { Versuchs- } \\
\text { Nummer }\end{array}$ & $\begin{array}{c}\text { Gesamtstickstoff } \\
\text { in } 10 \mathrm{~g} \text { fenchten } \\
\text { Leberbreies } \\
\mathrm{g}\end{array}$ & $\begin{array}{l}\text { Unkoagulabler Stick- } \\
\text { stoff in } 10 \mathrm{~g} \\
\text { Leberbrei } \\
\mathrm{g}\end{array}$ & 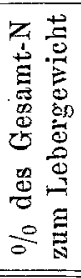 & 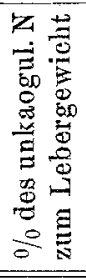 & 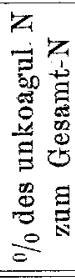 & 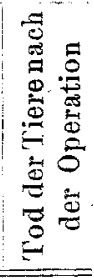 \\
\hline 1 & 0,29865 & 0,02685 & 2,99 & 0,27 & 8,99 & 7 Tage \\
\hline 2 & 0,29800 & 0,02336 & 2,98 & 0,23 & 7,84 & $7 \%$ \\
\hline 3 & 0,35004 & 0,03110 & 3,50 & 0,31 & 8,88 & $8 \%$ \\
\hline 4 & 0,32848 & 0,03005 & 3,28 & 0,30 & 9,15 & $9 \gg$ \\
\hline Ourchsehn.: & $227,71 \mathrm{cem} \cdot \frac{\mathrm{N}}{10} \mathrm{H}_{2} \mathrm{SO}_{4}$ & $19,89 \mathrm{ccm} \cdot \frac{\mathrm{N}}{10} \mathrm{H}_{2} \mathrm{SO}_{4}$ & 3,19 & 0,28 & 8,72 & \\
\hline
\end{tabular}

Tabelle 12.

Untersuchung der Leber der nach der Entmilzung sensibilisierten Meerschweinchen.

\begin{tabular}{|c|c|c|c|c|c|c|c|}
\hline $\begin{array}{c}\text { Vers.- } \\
\text { Nr. }\end{array}$ & 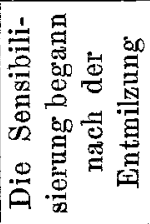 & 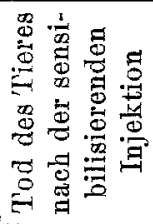 & 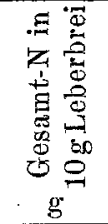 & 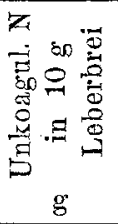 & 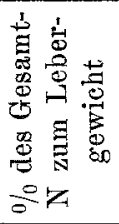 & 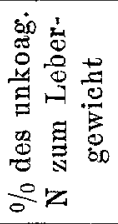 & 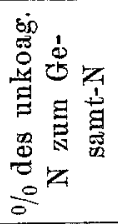 \\
\hline 1 & $1 \mathrm{Tag}$ & 14 Tage & 0,34968 & 0,03028 & 3,50 & 0,30 & $8,66$. \\
\hline 2 & 1 & 15 & 0,32165 & 0,02513 & 3,22 & $0,2 \tilde{z}$ & 7,81 \\
\hline 3 & 1 & 16 & 0,25589 & 0,02207 & 2,56 & 0,22 & 8,23 \\
\hline 4 & 1 & $\Rightarrow *$ & 0,33081 & 0,02909 & 3,31 & 0,29 & 8,79 \\
\hline 5 & 1 & $\gg *$ & 0,32782 & 0,02974 & 3,28 & 0,30 & 9,07 \\
\hline 6 & 15 Tage & $\gg *$ & 0,35175 & 0,04620 & 3,52 & 0,46 & 13,13 \\
\hline 7 & 15 & 14 & 0,31292 & 0,03867 & 3,13 & 0,39 & 12,36 \\
\hline 8 & 15 & 16 & 0,31011 & 0,03962 & 3,10 & 0,40 & 12,78 \\
\hline 9 & 17 & 14 & 0,23584 & 0,02879 & 2,36 & 0,29 & 12,21 \\
\hline
\end{tabular}

In den mit * bezeichneten Versuchen wurden die Tiere durch anaphylaktisehen Shoek, in den anderen durch Entbluten getötet.

kennen gelernt haben; während bei letzteren das Prozentverhältnis des unkoagulablen zum Gesamt- $\mathrm{N}$ im Mittel 21,98\% betrug, finden wir im Mittel den Prozentgehalt des ankoagulablen Leberstickstoffs der vier entmilzten Tiere mit 12,62\%. Immerhin ist hier eine geringe Steigerung des Leberabbaues gegenubber den fünf vorher er- 
Über d. intravitalen Eiweißabbau in d. Leber sensibilisierter Tiere usw, 109

wähnten Tieren, bei denen die Milz etwa 15 Tage vor dem Tode entfernt worden war, bemerkenswert, so daß der Schluß gezogen werden maß, daß nach 30 Tagen der Organismus die dureh den Ausfall der Milzfunktion gesetzten Folgen zum Teil bereits überwunden hat und andere Organsysteme vikariierend die Tätigkeit der Milz übernahmen $\left.{ }^{1}\right)$. Aus allen diesen Versuchen geht jedoch ubereinstimmend die wichtige Tatsache hervor, dab der Milz für den intravitalen Leberabbau der sensibilisierten Tiere eine maßgebende Rolle zufällt.

b) Milzexstirpation bei sensibilisierten Tieren.

War in den eben erwähnten Versuchen der Einfluß der Milz auf den Leberabbau bei nachfolgender Sensibilisierung gezeigt worden, so mußte geprïft werden, ob anch im Verlaufe der Überempfindlichkeitsperiode, zur Zeit, wo die intravitale Leberautolyse sich bereits entwickelt hatte, die Entfernung der Milz den Eiweißabbau in der Leber irgendwie $z \mathfrak{u}$ beeinflussen vermochte. Zu diesem Zweek wurde sensibilisierten Meerschweinchen 14 Tage nach der Seruminjektion die Milz entfernt und die Tiere 6 Tage später durch Verbluten aus der Carotis getötet. Die in folgender Tabelle an-

Tabelle 13.

Untersuchung der Leber der nach der Sensibilisierung entmilzten Meerschweinchen.

\begin{tabular}{|c|c|c|c|c|c|c|c|}
\hline $\begin{array}{l}\text { Vers.- } \\
\text { Nr. }\end{array}$ & 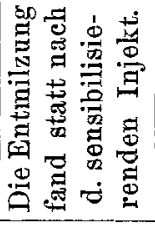 & 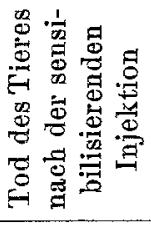 & 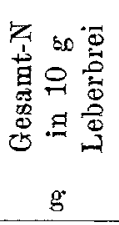 & 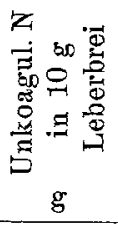 & 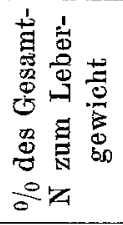 & 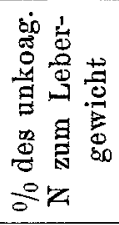 & 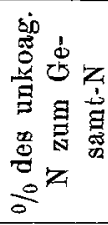 \\
\hline 1 & & & & & & $0, i$ & \\
\hline 2 & & & 0,31280 & 0,03283 & 3,13 & 0,33 & 10,50 \\
\hline
\end{tabular}

gefuihrten Werte zweier derart vorbehandelter Meerschweinchen lassen auf das deutlichste ersehen, daß unter dem Einfluß der Milzexstirpation selbst die intensive intravitale Leberantolyse hoch sensibilisierter Tiere sich innerhalb von 6 Tagen nahezu völlig rückbilden kann; das Verhältnis des inkoagulablen Leberstickstoffs zum

1) Einen :ihnlichen, allmählich in der 3.-4. Woche eintretenden Ersatz der Milzfunktion konnten auch Joannovics und Pick (a. a. 0.) an der durch Milzexstirpation gestörten Oxydation der Leberfette beobachten. 
Gesamtstickstoff beträgt in diesen Fällen $\mathbf{9 , 9 8}$ und 10,50\%, während, wie die fruheren Versuche zeigen, 20 Tage nach der Eiweißinjektion getötete, nicht operierte Meerschweinchen etwa $\mathbf{2 0} \%$ ihres Leberstickstoffs in unkoagulabler Form enthalten. Diese Versuche beweisen, daß nicht allein für das Entstehen der intravitalen Leberautolyse, sondern auch fï r den weiteren Bestand derselben die Milz von ausschlaggebender Bedentung ist; es mussen demnach in der Milz Stoffe produziert werden, welche den proteolytischen Abbau in der Leber fördern. Ob die Produktion dieser "Milzaktivatoren" schon unter physiologischen Bedingungen eintritt, muß dahingestellt bleiben; daß jedoch unter bestimmten pathologischen Verhältnissen, wie im Falle der Eiweißsensibilisierung, derartige intime Beziehungen zwischen Milz und Leber sich ausbilden und bestehen, zeigen einwandfrei die angefiihrien Versuche. Gerade die mächtige intravitale Beeinflussung des Leberabbaues durch die Milz unter gewissen pathologischen Bedingungen scheint uns von größter Bedeutung für manche Lebererkrankungen zu sein, und es lieg't wohl am nächsten das rätselhafte Krankheitsbild der akuten gelben Leberatrophie mit unseren experimentellen Befunden in Beziehung zu bringen. Es ist nicht unwahrscheinlich, daB bei dieser mit intensivem Leberabbau einhergehenden schweren Erkrankung die Tätigkeit der Milz, zumal der pathologisch veränderten Milz, von ausschlaggebender Bedeutung in der Richtung ist, daß der in der Norm nur in engen Grenzen sich vollziehende Zellabbau in der Leber nunmehr in exzessiver Weise gesteigert wird. Es könnte demnach bei Lebererkrankungen dieser Art in der veränderten Milzfunktion die primäre Ursache der Leberveränderung gesucht werden. Daß in der Tat manche Lebererkrankungen, insbesondere die akute gelbe Leberatrophie, im Gefolge schwerer Anämien eintreten, ist eine häufig gemachte klinische Beobachtung; die in jüngster Zeit von Eppinger1), allerdings aus anderen Grïnden, bei gewissen Lebererkrankungen vorgeschlagenen und mit gutem Erfolg. durchgeführten Splenektomien wưrden eine wertvolle Ergänzung. der von uns an Tieren gewonnenen experimentellen Erfabrungen bieten.

Das Fehlen der intravitalen Leberautololyse bei sensibilisierten Meerschweinchen nach vorausgegangener Milzexstirpation hätte auch dadurch erklärt werden können, daß sich infolge der Splenektomie überhaupt keine oder nur sehr spärliche Immunkörper gebildet hätten,

1) a. a. 0 . 
Über d. intravitalen Eiweißabbau in d. Leber sensibilisierter Tiere nSw. 111

da seit den Untersuchungen von R. Pfeiffer und Marxi) die blutbereitenden Organe als Hauptbildungsstätten der Antikörper angesehen werden. Da in unseren Versuchen nur die Milz entfernt worden war, Knochenmark und Lymphdrüsen jedoch intakt geblieben waren, war es an sich wenig wahrscheinlich, daß die Immunkörperbildung einen wesentlichen Schaden erlitten hatte, zumal anderweitige vielfache Beweise für die Produktion der Antikörper in den verschiedensten Organzellen existieren. In unserem Falle lieb sich in der Tat leicht nachweisen, daß trotz der Splenektomie sowohl die einen Tag; als auch 14 Tage später vorgenommene Sensibilisierung zur Produktion von anaphylaktischen Immunkörpern geführt hat; denn Tiere dieser Art gingen auf der Höhe der Sensibilisierung, wie aus den angeführten Tabellen za ersehen ist (Tabelle 12), bei der intravenösen Reinjektion mit 1,5-2 ccm Pferdeserum unter denselben typischen anaphylaktischen Symptomen wie nicht splenektomierte Tiere zugrunde. Andererseits zeigten auch jene Tiere, bei denen die Milz erst 14 Tage nach der Eiweißinjektion exstirpiert worden war, also bei völlig ungestörter Immunkörperbildung, schon 6 Tage nach der Operation in bezug auf die Stickstoffverteilung in der Leber dieselben der Norm entsprechenden Verhältnisse wie die nach der Milzexstirpation sensibilisierten. Aus diesen Tatsachen geht hervor, daß nicht etwa der Mangel der Immunkörperbildung nach der Milzexstirpation die Ursache für das Ausbleiben der intravitalen Autolyse sein kann, daß vielmehr durch Entfernung der Milz andere Funktionen dieses Organes ausgeschaltet wurden, die unter dem Einfluß der Eiweißrorbehandlung entweder entstehen oder gesteigert werden. Die Entstehung der Immankörper und die Aktivierung des intravitalen Leberabbaues durch die Milz sind demnach zwei von der parenteralen Eiweißzufuhr wohl abhängige, jedoch voneinander unabhängig verlaufende Prozesse; ebenso steht auch der durch Bronchialkrampf erzeugte anaphylaktische Tod der Meerschweinchen nicht in ursächlicher Beziehung zu der intravitalen Leberautolyse.

\section{Zusammenfassung.}

Die eben mitgeteilten Versuche decken wichtige Beziehungen auf, welche zwischen parenteraler Einverleibung artfremden Eiweißes und proteolytischem Abbau in den lebenden Organzellen, speziell in der Leber, bestehen. Die bisherigen Untersuchungen, die wir vorwiegend

1) Pfeiffer, R. und Marx, Zeitsehr. f. Hygiene a. Infekt. Bd. 27, 1898. 
Abderhalden, Heilner und deren Mitarbeitern verdanken, hatten festgestellt, daß nach parenteraler Einfuhr »blatfremder " Eiweißkörper bei Mensch und Tier proteolytische Fermente, sogenannte "Schutz-, Abwehr- oder Notfermentes in der Blutbahn erscheinen, welche auBerhalb des Organismus, auf totes nnd denaturiertes Eiweißmaterial eine mäßige Wirkung zu entfalten vermögen. Ob derartigen Fermenten auch im lebenden Organismus irgendein Einfluß zukommt, blieb nach den vorliegenden Ergebnissen durchaus zweifelhaft; denn der Befund von Biuretreaktion liefernden Eiweißabbauprodukten, wie er im Blute sensibilisierter und anaphylaktischer Tiere von manchen Autoren erhoben wurde, kann um so weniger Beweiskraft für die Existenz eines gesteigerten intravitalen Organabbaues beanspruchen, als es sich hierbei stets nur um eine qualitative, die äußersten Spuren von Eiweißspaltprodukten kennzeichnende Reaktion handelt; zudem haben zahlreiche neuere Untersuchungen (Folin, v. Slyke, Abderbalden, Abel und ihre Mitarbeiter) schon unter normalen Verhältnissen in Übereinstimmung mit älteren Angaben (E. Freund) Eiweißspaltprodukte in der Blutbahn nachweisen können. Es muß daher zugestanden werden, daß trotz des hohen wissenschaftlichen Interesses, den der Befund von proteolytischen Fermenten im Blutserum eiweißrorbehandelter Tiere hatte, bisher jeder sichere Anhaltspunkt sowohl für die intravitale Bedentung dieser Fermente, als auch für das Bestehen einer intravitalen Organantolyse fehlte. Die von uns durchgefiuhrten Untersuchungen zeigen, daß in der Tat durch einmalige parenterale Applikation äuBerstgeringer Mengen körperfremden Eiweißes eine gewaltige Organproteolyse im Tierkörper einsetzt, die vorwiegend die Leber betrifft, so daB 1/s-1/4 des Lebereiweißes in spaltprodukte umgewandelt wird; der Umstand, daß alle anderen untersuchten $\mathrm{Or}$ gane sowie das Blut gegenüber der Leber in ihrem Abbaurermögen bedeutend zurücktreten, weist darauf hin, daß bei der gewählten Vorbehandlung (subkutane Injektion von Pferdeserum) vorwiegend in den Leberzellen die Produktion oder Aktivierung dieser anf den spezifischen Leberzellenabbau eingestellten Fermente stattfindet. Diese Annahme besitzt einen hohen Grad von Wahrscheinlichkeit und läßt sich, wie in einer weiteren Mitteilnng gezeigt werden soll, auch experimentell begründen. Sie findet außerdem in der Tatsache eine wichtige Stuitze, dah im lebenden Organismus die Anwesenheit der Milz für die Aktivierung dieser autolytischen Leberprozesse nötig ist, die also trotz Anwesenheit ron Antikörpern im Blutkreislauf beim Fehlen dieser Milzaktivatoren nicht 
Über d. intravitalen Eiweißabbau in d. Leber sensibilisierter Tiere usw. 113

oder nur in geringfiigigem Umfange eingeleitet werden können. Auch die alle anderen Organe schonende, streng auf die Leberzellen eingestellte spezifische Fermentwirkung ist für die zelluläre Abstammung dieser autolytischen Fermente bezeichnend. Es scheint aber auch der Schluß nicht unwahrscheinlich, daB auch die in der Blutbahn gefundenen proteolytischen Fermente vorwiegend oder ansschließlich den Organzellen, in unserem Falle den Leberzellen, entstammen und nur einen in die Blutbahn ausgeschwemmten geringen Fermentrest darstellen, von dem es fraglich bleibt, ob ihm eine größere Bedeutung für den intravitalen Abbau iberhaupt zukommt; der Hauptanteil an der intravitalen Leberautolyse scheint zweifellos den in den Leberzellen selbst aktivierten Fermenten zuzukommen.

Die an Hefezellen ausgeführten Untersuchungen Rubnersi) über die Beteiligung endozellulärer Fermente am Energieverbranch der Zelle bilden eine interssante Analogie zu diesen eben erörterten Verbältnissen, indem auch aus den Experimenten Rubner's hervorgeht, daß die nach Buchner aus der Hefe dargestellten, von der Zelle abtrennbaren Fermente nur etwa 1,6-4,6\% der Gesamtgärleistung der Zelle bilden, so daß 95,4-98,4\% der Gesamtleistung den zellulären Vorgängen zugeschrieben werden müssen. Ähnliche Größenverhältnisse fand auch Warburg ${ }^{2}$ ) bei Vergleich der Sauerstoffatmung der aus Sängetierlebern hergestellten, durch Berkefeldkerzen filtrierten Extrakte und des intakten Lebergewebes; die Filtratatmung betrug nur etwa 4\% der Zellatmung. Es dürfte daher die Überlegenheit der zellalären Fermentleistungen gegeniiber den extrazellulären für die verschiedensten Fermente Gültigkeit besitzen.

Die merkwuirdige Erscheinung, daß nur in der Leber so ausgedehnte autolytische Prozesse bei der Vorbehandlnng der Meerschweinchen mit Pferdeserumeiweiß eingeleitet werden, könnte zunächst in Zusammenhang danit gebracht werden, daß diese im Mittelpunkt des Gesamtstoffwechsels stehende wichtigste Drüse des tierischen Organismus bei der Verarbeitung des parenteral zugefuihrten körperfremden Kolloids von allen Organzellen am meisten in Mitleidenschaft gezogen wird. Dieser Vorstellung steht jedoch die Er-

1) Rubner, M., Über die Beteiligung endozellularer Fermente am Energieverbranch der Zelle. Sitzungsberichte der Königl. Preußischen Akademie der Wissensch. Sitzg. d. physik.-math. Klasse v. 1. Februar 1912, S. 124.

2) Warburg, 0., Über sauerstoffatmende Körnchen aus Leberzellen und über Sauerstoffatmung in Berkefeldfiltraten wässeriger Leberextrakte. Pflügers Archiv Bd. 154, S. 599, 1913. 
wägung entgegen, daß durch die Eiweißsensibilisierung zweifellos auch viele andere, wenn nicht alle Organzellen, ja sogar Nervenzellen, trotz ihres höchst torpiden Stoffiwechsels, in eingreifendster Weise derart beeinflußt werden, daß ihre funktionelle Reaktionsfähigkeit eine auffällige quantitative Änderung erfährt. Wir müssen uns daher eher der Ansicht zuneigen, daB die Aktivierung der Autolyse in den Leberzellen sensibilisierter Tiere in Parallele zu setzen ist mit den an überempfindlichen Meerschweinchen beobachteten Erscheinnngen in anderen Zellgebieten, wie etwa mit der Übererregbarkeit der glatten Uterus-, Darm- und Bronchialmuskulatux oder mit der Übererregbarkeit der Nervenendigungen des Lungenvagus. Die gesteigerte Autolyse der Leberzellen wäre danach nichts anderes als der Ausdruck der unter dem Einfluß des Antigens auf bisher noch unbekannte Weise gesteigerten spezifischen Funktion der Leberzellen, die jedoch in keinem direkten Zusammenhange mit der Verarbeitung des parenteral zugefuhrten artfremden Eiweißkolloids steht. Daß gerade die Leberzelle mit gesteigerter Autolyse reagiert, ist wohl einerseits in ihrer spezifischen Fanktion, andererseits in dem innigen Zusammenhange der autolytischen Fermente mit dem Zellprotoplasma begriundet. Gerade die pathologisehen Prozesse bieten ein gutes Beispiel für diese offenbar keinem anderen Organe so sehr wie der Leber spezifisch zugehörige, in gesteigerter Autolyse sich ausdrickende Reaktionsqualität ihrer Zellen; die autolytische Einschmelzung des Organs bei der Phosphorvergiftung, der akuten gelben Leberatrophie und manchen Cirrhosen, der Chloroformvergiftung bezeugen zur Genïge, daß auf die verschiedensten Reize die Leberzelle mit derselben Steigerung ihrer fermentativen Zellfunktion antwortet.

Diese Auffassung der gesteigerten Leberantolyse sensibilisierter Tiere kann auch die Erklärung dafür abgeben, daß die anderen Organe keinen merklich gesteigerten Zellabbau aufweisen. Es wäre eben möglich, daß auch sie im Sinne ihrer spezifischen Zellfunktion auf die Folgezustande der Antigenzufuhr reagieren; diese Änderungen der Zellfunktion müssen dabei jedoch durchaus nicht auf dem Gebiete des antolytischen Abbanes gelegen sein; sie änßern sich möglicherweise in Reaktionen, die uns vorläufig unbekannt sind oder sich mit den uns zugänglichen Methoden noch nicht geü̈gend quantitativ beurteilen lassen. Bei dieser Betrachtungsweise mag es jedoch offen gelassen werden, ob nicht bei Verwendung eines anderen Antigens, als des von uns benützten Pferdeserums, etwa bei parenteraler $\mathrm{Zufuhr}$ ron Organeiweiß, anch andere Organe als die Leber spezifischen autolytisehen Abbanes fähig sind, eine Frage, die weiteren Studiums bedarf. 
Die sinnfälligste Tatsache, welche durch die vorliegenden Studien festgestellt werden konnte, ist wohl der bedeutende Umfang der intravital einsetzenden Organautolyse; die subkutane Injektion von weniger als $0,0001 \mathrm{~g}$ Serumeiweiß genïgt, um einen Leberzerfall anzuregen, der nabezu $1 / 4$ des Organs ergreift. Die große quantitative Differenz, welche zwischen der Menge des behufs Sensibilisierung eingeführten und des zerfallenen Eiweißes besteht - die abgebaute Eiweißmenge ist in unsereren Fällen etwa 3000-5000mal größer als die eingeführte, ein Verhältnis, das bei entsprechender Versuchsanordnung noch augenfälliger gestaltet werden kann -, gestattet mit voller Sicherheit den Schluß, daß das abgebaute Eiweiß nahezu ausschließlich körpereigenes Eiweiß ist und daß der Abbau des eingeführten körper- oder blutfremden Eiweißes daneben gar nicht in Frage kommt. Aus dieser Feststellnng ergibt sich, daß diese bei der intravitalen Leberautolyse tätigen und durch die parenterale Eiweißzufuhr aktivierten Fermente nicht Schutz- und Abwehrfermente im Sinne der Hypothese $A b$ derhaldens sein können, nach welcher das Auftreten von proteolytischen Fermenten nach parenteraler Eiweißzufuhr einer rom Organismus eingeleiteten Schutzrorrichtung entspricht, welehe die ausgeschaltete Magen-Darmverdauung intermediär ersetzen und das als Gift wirkende körperfremde Eiweiß der Assimilation zugänglich machen soll; die von Abderhalden, Pincussohn u. a. im Blute immunisierter Tiere nachgewiesenen, spezifisch gegen das zugefuhrte Antigen gerichteten Fermente miissen daher von den von uns nachgewiesenen autolytischen Leberfermenten bis auf weiteres getrennt werden.

Der hauptsächlich in der Leber sich abspielende autolytische Zerfallsprozeß bietet gleichzeitig eine Erklärung dafür, daß wir in der Blutbahn sensibilisierter Tiere kaum eine Änderung im Bestande der stickstoff haltigen Anteile gegenüber der Norm nachweisen konnten. Denn wir können hier im besten Falle nur einen geringen, während der Resorption der zerfallenen Organzellen in Lymphe und Blnt gelangten Teil der in den Leberzellen angehäuften Abbauprodukte antreffen; da diese Spaltprodukte zum allergrößten Teil rasch aus der Blutbahn durch die Nieren ausgeschieden werden, ist es verständlich, daß unter den gegebenen Bedingungen selbst eine voriibergehende Vermehrung der normalerweise vorkommenden EiweiBspaltprodukte im Blute sich dem Nachweis entziehen könnte.

Der Nachweis von Eiweißabbaukrodukten in den Organen sensibilisierter Tiere könnte Anlaß geben, denselben als Stuitze für die vielfach (H. Pfeiffer, Biedl und Kraus, Abderhalden, Heilner) 
geäußerte Ansicht heranzuziehen, daß der anaphylaktische Shock sensibilisierter Tiere in ursächlichem Zusammenhange mit dem durch das Antigen erzeugten Eiweißabbau stünde. Wiewobl in unseren Untersuchungen gerade der Nachweis von Eiweißspaltprodukten in der Blutbahn, der bisher als pathognomonisch bei den Verfechtern der humoralen Entstehung der giftigen Spaltprodukte postuliert wurde, iuberhaupt nicht gelang, wäre die Möglichkeit gegeben, daB den in den Organen fixierten Abbauprodukten die gleiche Rolle zufällt. Die Beobachtung an sensibilisierten Tieren, bei welchen die intravitale Leberautolyse maximal entwickelt ist, ergibt indessen keinerlei Anhaltspunkte für das Vorhandensein einer toxischen Wirkung der aus dem Zerfall der Leberzellen entstandenen Spaltprodukte; die Tiere zeigen während der Sensibilisierungsperiode ein durchaus normales Verhalten 1). Aber gerade der durch unsere Versuche erbrachte Nachweis, daß der Ausbruch des anaphylaktischen Shocks in gleicher Weise bei splenektomierten Meerschweinchen, die keinerlei Erscheinungen von Leberzerfall zeigen, zu erzeugen ist, wie bei normalen sensibilisierten Tieren mit mächtiger Leberantolyse, spricht dafuir, daß die beobachtete Organautolyse und deren Produkte für das Auftreten der Anaphylaxie nicht verantwortlich gemacht werden können; es ist daher die unter der Antigenwirkung sich allmählich und mit den funktionellen Veränderungen an anderen Organen gleichzeitig entwickelnde Leberautolyse als ein selbständiges, den Erscheinungen an den iibrigen Zellen gleichgeordnetes Phänomen anzusehen. Die durch die intravitale Leberantolyse freigewordenen Eiweißzersetzungsprodukte können daher nicht bei Meerschweinchen die Ursache für die Auslosung des anaphylaktischen Bronchospasmus abgeben, der vielmehr ebenfalls nur als eine selbständige, von den anderen Organzellenänderungen unabhängige, spezifische Funktionsänderung der glatten Bronchialmuskelzellen und der zugehörigen autonomen Nervenelemente aufgefaßt wer-

1) Die gefundene Anreicherung stickstoffhaltiger Spaltprodukte in der Leber könnte wohl auch durch eine Hemmung des normalerweise stattindenden weiteren Abbaues derselben während der Sensibilisierungsperiode eine Erklärung fnden; fiir eine solche Annahme fehlt jedoch vorläufig ein Anhaltspunkt, zumal sich in anderweitig mitgeteilten Versnchen nachweisen ließ, daß auch am ïberlebenden Leberbrei normaler Meerschweinchen drurch Sensibilisierung eine be. deutende Steigerung des autolytischen Leberzerfalls erzengt werden kann. (Siehe E.P.Pick und M. Hashimoto: Sensibilisierung und anaphylaktischer Shock der iberlebenden Meerschweinchenleber: Zeitschr. f. Immunitätsforschnng $n$. exper. Therapie Bd. 21. S. 237, 1914.' 
den muB. Die gewöhnliche, durch Bronchialkrampf bedingte Todesursache der anaphylaktischen Meerschweinchen stellt sich nach dieser Auffassung als eine mehr zufällige und augenfällige dar; daß auch schwere andersartige, von der Erstickung durch Bronchialkrampf durchaus unabhängige funktionelle Änderungen' in den tibrigen Organen durch den anaphylaktischen Shock ansgelöst werden können, soll noch in einer weiteren Mitteilung erörtert werden.

Daß jedoch der Leber bei manchen Tierarten für das gesamte Vergiftungsbild eine sehr wichtige Rolle zufällt, geht schon aus den eingangs erwähnten und mehrfach bestätigten Versuchen Manwarings hervor, der nach Leberausschaltung bei Hunden den anaphylaktischen Shock ausbleiben sah. Wir wissen ferner, insbesondere aus den Untersuchungen von Biedl und Kraus, daß gerade beim Hunde im Gegensatz zum Meerschweinchen der anaphylaktische Symptomenkomplex sich hauptsächlich im Gebiete der Baucheingeweide (Splanchnicusgebiet) abspielt. Die mächtige Leberveränderung während der Sensibilisierungsperiode liefert den Beweis fuir die intensive Beteiligung der Leberzellen an den sich abspielenden anaphylaktischen Reaktionen, und es ist daher leicht begreiflich, daß mit der kinstlichen Ausschaltung dieser gerade für die Erscheinungen der Anaphylaxie beim Hunde wichtigen Drüse aus dem allgemeinen Kreislauf ein sehr großer Teil höchst reaktionsfähiger Organzellen für die Erzeugung der schweren Vergiftung ungeeignet wird und dadurch der anaphylaktische Shock entweder völlig ausbleibt oder mitigierter abläuft; auf diese Weise stïnden beim Hunde die Leberveränderungen im Mittelpunkt der anaphylaktischen Vergiftung. 